\title{
甲硅烷基(硅烯)钉配合物中甲硅烷基甲氧基化反应机理的理论研究
}

\author{
周莉 ${ }^{a}$ 李 阳 ${ }^{a}$ 林芙蓉 ${ }^{a}$ 田迪英 ${ }^{a}$ \\ 雷群芳 $b$ 方文军 $b$ 谢湖均*, $a$ \\ ( ${ }^{a}$ 浙江工商大学应用化学系 杭州 310018) \\ ( ${ }^{b}$ 浙江大学化学系 杭州 310028)
}

\begin{abstract}
摘要 采用密度泛函理论(DFT), 我们研究了甲硅烷基(硅烯)钉配合物 $\mathrm{Cp} * \mathrm{Ru}(\mathrm{CO})\left(=\mathrm{SiMes}_{2}\right) \mathrm{SiMe}_{3}$ 与甲醇发生反应的 机理. 整个反应机理包含以下四个步骤: (1) $\mathrm{Ru}=\mathrm{Si}$ 双键和甲醇发生 1,2 加成反应, 随后通过消除 $\mathrm{Mes}_{2} \mathrm{Si}(\mathrm{OMe}) \mathrm{H}$ 生成 $\mathrm{Cp}^{*} \mathrm{Ru}(\mathrm{CO}) \mathrm{SiMe}_{3}$; (2)甲硅烷基配体上的甲基以 1,2 -甲基的迁移方式从硅原子转移到 $\mathrm{Ru}$ 原子上, 并与甲醇发生 1,2 加成 反应, 生成的甲烷脱离后产生 $\mathrm{Cp} * \mathrm{Ru}(\mathrm{CO}) \mathrm{Si}(\mathrm{OMe}) \mathrm{Me}_{2}$; (3)重复步骤(2)两次, 得到甲氧基化的产物 $\mathrm{Cp} * \mathrm{Ru}(\mathrm{CO}) \mathrm{Si}(\mathrm{OMe})_{3}$; (4)异腈配位 $\mathrm{Ru}$ 原子后得到最终的产物 $\mathrm{Cp} * \mathrm{Ru}(\mathrm{CO})(\mathrm{CN} t-\mathrm{Bu}) \mathrm{Si}(\mathrm{OMe})_{3}$. 甲醇和 $\mathrm{Ru}=\mathrm{Si}$ 双键的 1,2 加成是整个反应的决 速步骤, 自由能势垒为 $35.3 \mathrm{kcal} / \mathrm{mol}$. 此外, 还研究了甲硅烷基(锗烯)钓配合物与甲醇的反应机理, 相比硅烯配合物, 锗烯配合物决速步的能垒相对较低, 为 $31.8 \mathrm{kcal} / \mathrm{mol}$.
\end{abstract}

关键词 DFT 计算; 反应机理; 锗烯配合物; 硅烯配合物

\section{Reaction Mechanism for the Alkoxylation of a Silyl Ligand in the Silyl- (silylene)ruthenium Complex: A Density Functional Theory Study}

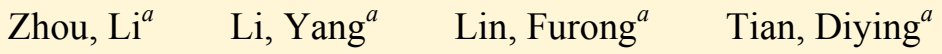 \\ Lei, Qunfang ${ }^{b} \quad$ Fang, Wenjun ${ }^{b} \quad$ Xie, Hujun ${ }^{*, a}$ \\ ( ${ }^{a}$ Department of Applied Chemistry, Zhejiang Gongshang University, Hangzhou 310018) \\ ( ${ }^{b}$ Department of Chemistry, Zhejiang University, Hangzhou 310028)
}

\begin{abstract}
The mechanism for the reaction of a silyl(silylene) ruthenium complex $\mathrm{Cp} * \mathrm{Ru}(\mathrm{CO})\left(=\mathrm{SiMes}_{2}\right) \mathrm{SiMe}_{3}$ with methanol was investigated via the density functional theory (DFT) calculations. The mechanism includes four steps. (1) The reaction initiates via the 1,2-addition of methanol to the $\mathrm{Ru}=\mathrm{Si}$ bond, followed by $\mathrm{Mes}_{2} \mathrm{Si}(\mathrm{OMe}) \mathrm{H}$ elimination to give $\mathrm{Cp} * \mathrm{Ru}(\mathrm{CO}) \mathrm{SiMe}_{3}$; (2) A methyl group of the silyl ligand migrates to $\mathrm{Ru}$ center via 1,2-Me migration, together with 1,2-addition of methanol, which is followed by the $\mathrm{CH}_{4}$ release to form $\mathrm{Cp} * \mathrm{Ru}(\mathrm{CO}) \mathrm{Si}(\mathrm{OMe}) \mathrm{Me}_{2}$; (3) Step (2) repeats twice to give the fully alkoxylated product $\mathrm{Cp} * \mathrm{Ru}(\mathrm{CO}) \mathrm{Si}(\mathrm{OMe})_{3}$. (4) Coordination of isonitrile affords final product $\mathrm{Cp} * \mathrm{Ru}(\mathrm{CO})\left(\mathrm{CN}^{t} \mathrm{Bu}\right) \mathrm{Si}(\mathrm{OMe})_{3}$. The first step involving the 1,2-addition of methanol to the $\mathrm{Ru}=\mathrm{Si}$ double bond is the rate-determining step for the whole reaction with a free energy barrier of $35.3 \mathrm{kcal} / \mathrm{mol}$. The mechanism for the reaction of a silyl(germylene)ruthenium complex with methanol was also considered. The rate-determining step has relatively lower barrier of $31.8 \mathrm{kcal} / \mathrm{mol}$ in contrast to the silyl(silylene) ruthenium complex.

Keywords DFT calculation; reaction mechanism; silylene complex; germylene complex
\end{abstract}

过渡金属卡宾配合物是金属有机化学的研究热点 之一, 近年来发展迅速, 而过渡金属硅烯配合物的发展 则相对缓慢. 在过去的几十年里, 许多从第 5 族到第 9 族的过渡金属硅烯配合物被合成和表征 ${ }^{[1]}$, 并被广泛地
应用于许多化学计量和催化反应过程中.

1987 年, Tilly 和同事 ${ }^{[2]}$ 合成了第一种乙腈配位的硅 烯钉配合物 $\left[\mathrm{Cp} *\left(\mathrm{Me}_{3} \mathrm{P}\right)_{2} \mathrm{Ru}=\mathrm{Si}(\mathrm{NCMe}) \mathrm{Ph}_{2}\right]\left[\mathrm{BPh}_{4}\right]\left(\mathrm{Cp}^{*}\right.$ $\left.=\eta^{5}-\mathrm{C}_{5} \mathrm{Me}_{5}\right)$, 并给出了晶体结构. 在 2007 年发表的一

*E-mail: hujunxie@gmail.com

Received October 16, 2014; revised December 10, 2014; published online January 5, 2015.

Project supported by the National Natural Science Foundation of China (No. 21203166) and the Food Science and Engineering of Most Important Discipline of Zhejiang Province (No. JYTsp2014111).

国家自然科学基金(No. 21203166)和食品科学与工程浙江省重中之重一级学科开放基金(No. JYTsp2014111)资助项目. 
篇综述文章中，他们 ${ }^{[3]}$ 讨论了采用三种通用的路线来合 成多种硅烯配合物, 这三种路线都包含阴离子基团提 取、游离硅烯的配位和 $\alpha$-氢的迁移步骤. 随着硅烯配合 物制备方法的不断发展, 对其化学反应活性的研究已经 成为可能 ${ }^{[4]} .1988$ 年, Ogino 等 ${ }^{[5]}$ 首次报道了给体一稳定的 二硅烯铁配合物. 在引入烷氧基后, 配合物表现出对空 气和湿度极高的敏感度. 他们还利用光化学和热化学方 法制备了过渡金属硅烯配合物. 反应活性研究表明, 硅 烯和甲硅烷基硅烯配合物中的 $\mathrm{M}=\mathrm{Si}$ 双键显示了强极 化 $\mathrm{Si}^{\delta+}=\mathrm{M}^{\delta-[6]}$. Tobita 等 ${ }^{[7]}$ 报道了一种中性无碱基稳定 的硅烯配合物 $\mathrm{Cp}(\mathrm{CO})_{2}(\mathrm{H}) \mathrm{W}=\mathrm{Si}(\mathrm{H})\left[\mathrm{C}\left(\mathrm{SiMe}_{3}\right)_{3}\right](\mathrm{Cp}=$ $\left.\eta^{5}-\mathrm{C}_{5} \mathrm{Me}_{5} ; \eta^{5}-\mathrm{C}_{5} \mathrm{Me}_{4} \mathrm{Et}\right)$ 的合成和表征, 这种配合物显示 了氢负配体和硅烯配体之间的相互作用. 实验还表明这 种配合物可以与不饱和小分子反应, 例如腈 ${ }^{[8]}$ 和甲基乙 烯基酮 ${ }^{[9]}$. 他们 ${ }^{[10]}$ 还研究了中性硅烯钌配合物 $\mathrm{Cp}^{*}(\mathrm{CO})(\mathrm{H}) \mathrm{Ru}=\mathrm{Si}(\mathrm{H})\left\{\mathrm{C}\left(\mathrm{SiMe}_{3}\right)_{3}\right\}$ 与异氧酸酯 $(\mathrm{ArNCO}$ $(\mathrm{Ar}=\mathrm{Mes} ; \mathrm{Ph} ; \mathrm{Mes}=2,4,6$-trimethylphenyl $)$ 和异硫氰酸 酯(MesNCS)的反应. 当此中性硅烯钉配合物与异氧酸 酯反应时, 发生 $\mathrm{C}=\mathrm{O}$ 键的硅氢化反应生成含有 $\mathrm{Ru}-$ $\mathrm{Si}-\mathrm{O}-\mathrm{C}-\mathrm{N}$ 五元环的产物. 当中性硅烯钓配合物与 异硫氰酸酯反应则发生 $\mathrm{C}=\mathrm{S}$ 键的断裂. 我们课题组 ${ }^{[1]}$ 采用密度泛函理论(DFT)对此反应机理进行了系统研究. 计算结果表明, 异氰酸酯和异硫氰酸酯的不同反应活性 是由 $\mathrm{C}=\mathrm{O}$ 和 $\mathrm{C}=\mathrm{S}$ 不同的键强度和 $\pi$ 接受能力, 以及 氧原子和硫原子对硅烯配合物中硅原子中心不同程度 的亲和力造成的.

最近, Tobita 等 ${ }^{[12]}$ 通过 $\mathrm{Cp} * \mathrm{Ru}(\mathrm{CO})(\mathrm{py}) \mathrm{Me}(\mathrm{py}=$ pyridine)与 $\mathrm{HSiMe}_{2} \mathrm{SiMes}_{2} \mathrm{Me}$ 的反应, 合成了甲硅烷基 (硅烯)钉配合物 $\mathrm{Cp} * \mathrm{Ru}(\mathrm{CO})\left(=\mathrm{SiMes}_{2}\right) \mathrm{SiMe}_{3}$, 并研究了 此配合物和甲醇在室温下生成 $\mathrm{Cp} * \mathrm{Ru}(\mathrm{CO})$ $(\mathrm{CNR})\left[\mathrm{Si}(\mathrm{OMe})_{3}\right](\mathrm{R}=t-\mathrm{Bu}, \mathrm{Xyl})$ 的反应. 尽管实验已经 做出了重大贡献, 但是对其反应机理还是悬而未决的. 在本文中, 我们采用密度泛函理论对此反应的具体机理 进行了详细探索.

\section{1 结果与讨论}

\section{1 甲硅烷基(硅烯)钉配合物中甲硅烷基甲氧基化的 反应机理}

如图 1 所示, 计算得到的配合物 $\mathrm{Cp} * \mathrm{Ru}(\mathrm{CO})(=$ $\left.\mathrm{SiMes}_{2}\right) \mathrm{SiMe}_{3}(\mathbf{1})\left(\mathrm{Cp} *=\eta^{5}-\mathrm{C}_{5} \mathrm{Me}_{5}, \mathrm{Mes}=\right.$ mesityl $)$ 结构参 数与从 X 射线获得的晶体结构参数相一致 ${ }^{[12]}$, 这表明计 算中我们使用的理论方法是合理的. 在晶体结构中, 配 合物 1 中 $\mathrm{Ru}-\mathrm{Si}(1)$ 的键长为 $2.234 \AA$, 而计算得到的 $\mathrm{Ru}-\mathrm{Si}(1)$ 的键长为 $2.302 \AA$, 两者的距离均在 $\mathrm{Ru}=\mathrm{Si}$ 双 键键长的范围内 $(2.22 \sim 2.34 \AA)$.

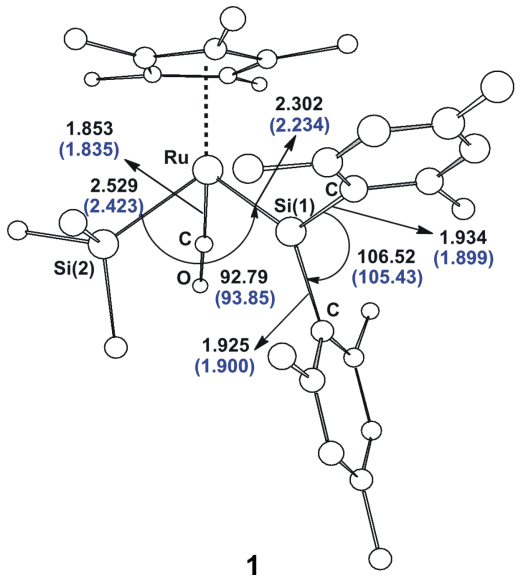

图 1 优化得到的配合物 1 的键长 $(\AA)$ 和键角 $\left(^{\circ}\right)$

Figure 1 Optimized structure with selected bond lengths $(\AA)$ and angles $\left({ }^{\circ}\right)$ for complex $\mathbf{1}$

The bond lengths and angles in parentheses are obtained from the crystal structure. The hydrogen atoms have been omitted for the purpose of clarity

图 2 显示了在异腈存在下, 一分子甲硅烷基(硅烯) 钉配合物 $\mathrm{Cp} * \mathrm{Ru}(\mathrm{CO})\left(=\mathrm{SiMes}_{2}\right) \mathrm{SiMe}_{3}(\mathbf{1})$ 与甲醇生成三 甲氧基甲硅烷基配合物 $\mathrm{Cp} * \mathrm{Ru}(\mathrm{CO})\left(\mathrm{CN}^{t} \mathrm{Bu}\right)\left[\mathrm{Si}(\mathrm{OMe})_{3}\right]$ 和 $\mathrm{Mes}_{2} \mathrm{Si}(\mathrm{OMe}) \mathrm{H}$. Tobita 等 ${ }^{[12]}$ 基于初步的实验结果, 提 出了一个可能的反应机理(图 3). 包括甲醇加成、还原 消除、3 次连续交替的 1,2-甲基迁移、甲醇 1,2 加成和 甲烷的释放步骤, 异腈配位得到烷氧基化产物. 在本文 中, 我们用 DFT 计算方法来详细地探讨具体的反应机 理.

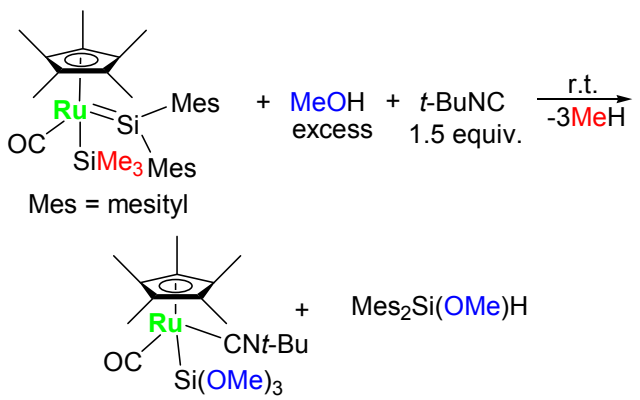

图 2 硅烯配合物 $\mathbf{1}$ 和甲醇的反应

Figure 2 The reaction of complex 1 with methanol

图 4 6 显示了硅烯配合物 1 与甲醇反应的自由能 势能面图, 对应化合物的结构参数如图 7 9 所示. 反应 机理可分为四个主要步骤: (1)甲醇和 $\mathrm{Ru}=\mathrm{Si}$ 双键的 1,2 加成, 随后发生 $\mathrm{Mes}_{2} \mathrm{Si}(\mathrm{OMe}) \mathrm{H}$ 的消除, 生成 $\mathrm{Cp} * \mathrm{Ru}-$ (CO) $\mathrm{SiMe}_{3}$ ；(2)甲硅烷基配体上的甲基以 1,2-甲基的迁 移方式从硅原子转移到 $\mathrm{Ru}$ 原子上, 再与甲醇发生 1,2 加 成反应，紧接着释放甲烷，并生成 $\mathrm{Cp} * \mathrm{Ru}(\mathrm{CO}) \mathrm{Si}(\mathrm{OMe})-$ $\mathrm{Me}_{2} ;$ （3)重复步骤(2)两次, 得到完全烷氧基化的产物 $\mathrm{Cp} * \mathrm{Ru}(\mathrm{CO}) \mathrm{Si}(\mathrm{OMe})_{3} ;$ （4)异腈配位后得到最终产物 

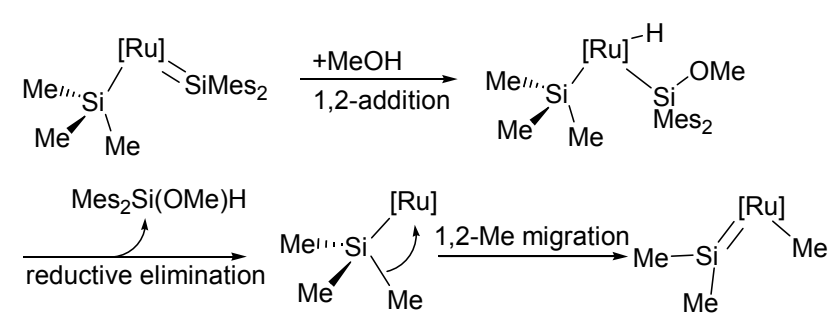

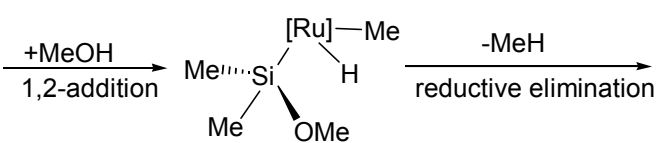

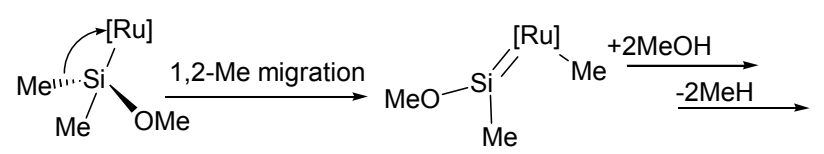

$[\mathrm{Ru}]=\mathrm{Cp}{ }^{*} \mathrm{Ru}(\mathrm{CO})$

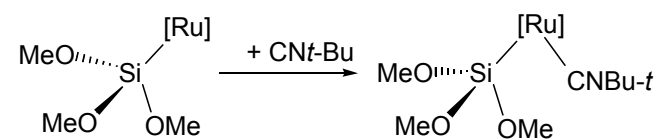

图 3 Tobita 和合作者提出的硅烯配合物 $\mathbf{1}$ 和甲醇反应的可能 机理

Figure 3 Possible mechanism for the reaction of complex 1 with methanol proposed by Tobita and co-workers

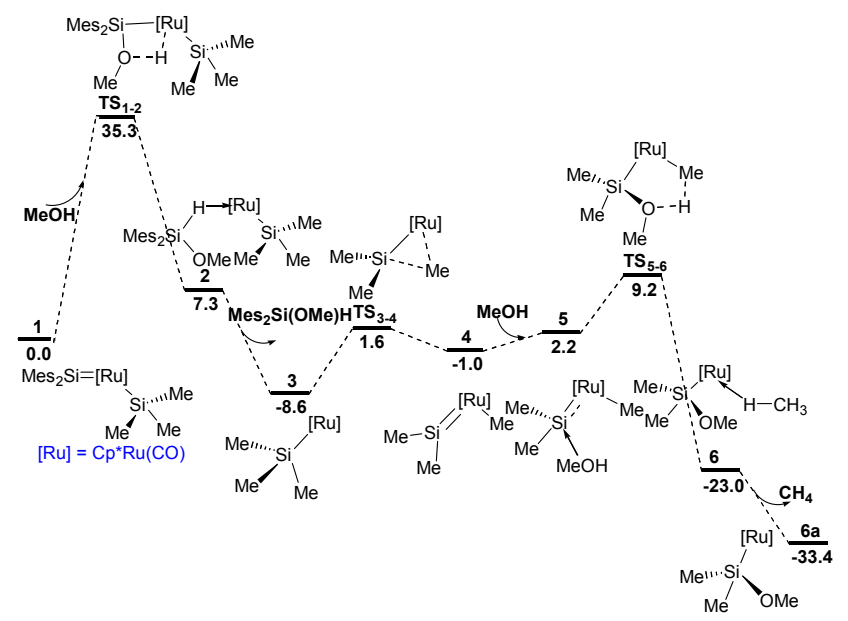

图 4 甲醇加成和甲硅烷基第一次甲氧基化的自由能势能面 (溶剂化自由能)

Figure 4 Free energy profiles of the methanol addition and first alkoxylation of the silyl ligand for the reaction of complex $\mathbf{1}$ with methanol

The solvation-corrected relative free energies are given in $\mathrm{kcal} / \mathrm{mol}$

\section{$\mathrm{Cp} * \mathrm{Ru}(\mathrm{CO})(\mathrm{CN} t-\mathrm{Bu}) \mathrm{Si}(\mathrm{OMe})_{3}$.}

首先, 我们研究了甲醇与 $\mathrm{Ru}=\mathrm{Si}$ 双键的 1,2 加成反 应. 如图 4 所示, 从配合物 $\mathbf{1}$ 出发, 甲醇中的氧原子配位 到硅原子上, 同时氢原子通过 1,2 加成的形式, 从氧原 子转移到钌原子上. IRC 计算表明, 配合物 2 中有一个抓 氢键. 1,2-加成反应需要克服 $35.3 \mathrm{kcal} / \mathrm{mol}$ 的自由能势 垒 $\left(\mathbf{T S}_{1-2}\right)$, 是整个多步烷氧基化反应的决速步骤. 计算 得到 $\mathbf{T S}_{1-2}$ 中 $\mathrm{Ru}-\mathrm{H}$ 和 $\mathrm{O}-\mathrm{H}$ 之间的距离分别为 1.844

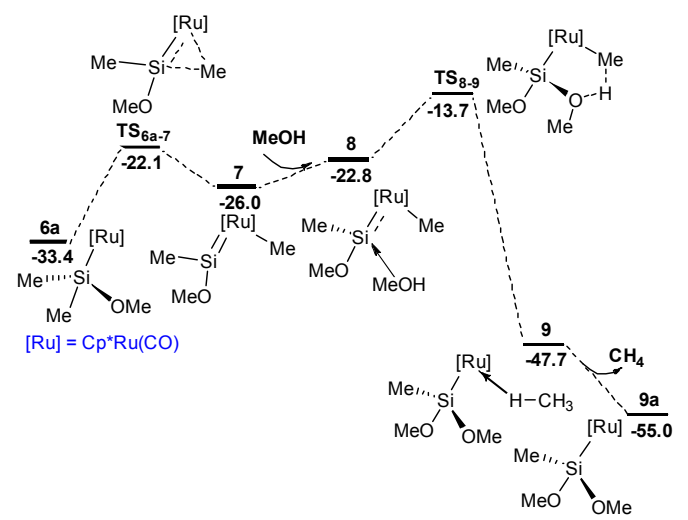

图 5 1,2-甲基迁移和甲硅烷基第二次甲氧基化的自由能势能 面(溶剂化自由能)

Figure 5 Free energy profiles of the 1,2-Me migration and second alkoxylation of the silyl ligand for the reaction of complex 1 with methanol

The solvation-corrected relative free energies are given in $\mathrm{kcal} / \mathrm{mol}$

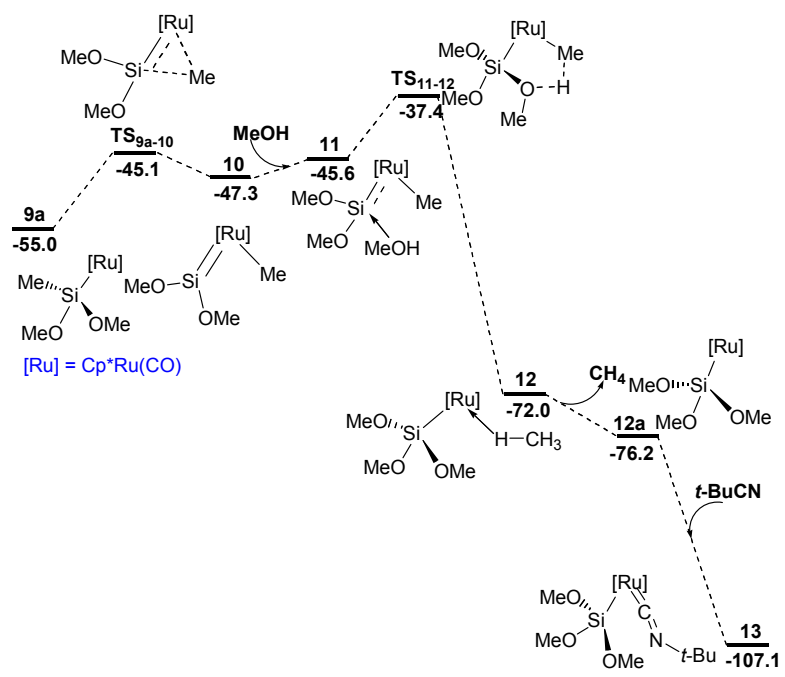

图 6 甲硅烷基第三次甲氧基化和异腈配位的自由能势能面 (溶剂化自由能)

Figure 6 Free energy profiles of the third alkoxylation of the silyl ligand and the coordination of isonitrile for the reaction of complex 1 with methanol

The solvation-corrected relative free energies are given in $\mathrm{kcal} / \mathrm{mol}$

和 $1.305 \AA$ (图 7). 随后, $\mathrm{Mes}_{2} \mathrm{Si}(\mathrm{OMe}) \mathrm{H}$ 从配合物 2 上脱 离生成配合物 3 , 并释放 $15.9 \mathrm{kcal} / \mathrm{mol}$ 的能量. 紧接着 发生 1,2-甲基转移反应, 需要克服的自由能势垒 $\left(\mathbf{T S}_{3-4}\right)$ 为 $10.2 \mathrm{kcal} / \mathrm{mol}$, 生成硅烯钉化合物 4. $\mathbf{T S}_{3-4}$ 中 $\mathrm{Ru}-\mathrm{Si}$, $\mathrm{Ru}-\mathrm{C}$ 和 $\mathrm{C}-\mathrm{Si}$ 之间的距离分别为 $2.266,2.252$ 和 2.572 $\AA$ (图 7). 之后, 另一分子的甲醇通过其上的氧原子配位 到配合物 4 中的硅原子上，生成配合物 $\mathbf{5}$, 同时氢原子 从甲醇的氧原子转移到与钉相连的甲基碳原子上. 这一 步需要自由能势垒 $\left(\mathbf{T S}_{5-6}\right.$ ) 为 $7.0 \mathrm{kcal} / \mathrm{mol}$ (从配合物 5 到 $\mathbf{T S}_{56}$ ). 接着，反应生成了含有抓氢键的中间产物 $\mathbf{6}$, 通 过释放 $10.4 \mathrm{kcal} / \mathrm{mol}$ 能量, 得到了甲氧基化中间产物 $6 \mathbf{a}$. 

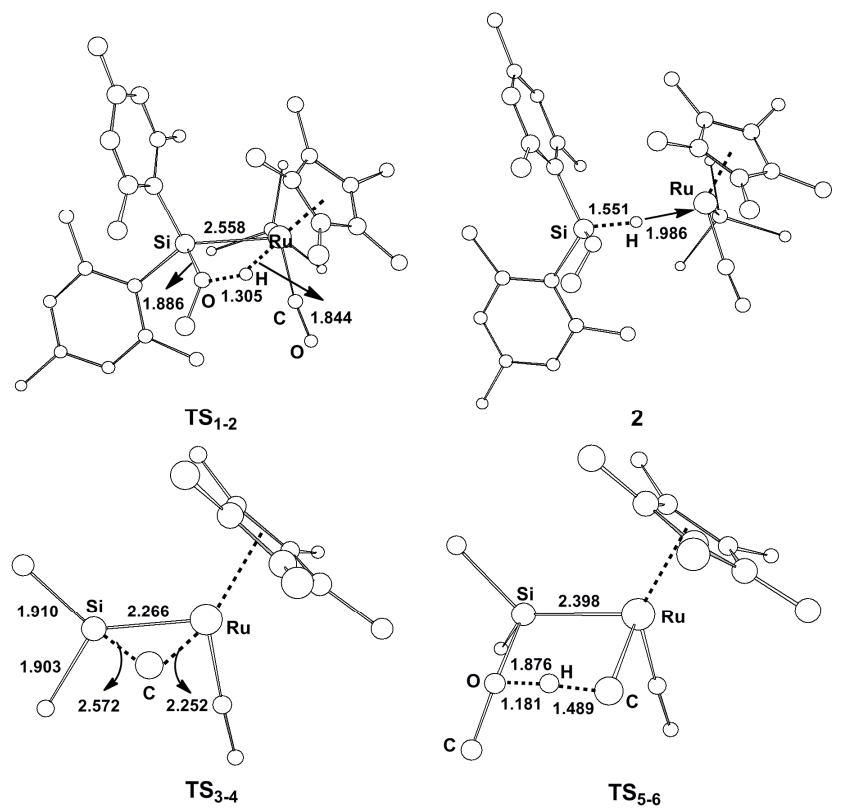

图 7 优化得到的如图 4 中所示的一些配合物的几何结构和键 长 $(\AA)$

Figure 7 Optimized structures with selected bond lengths $(\AA)$ for the species shown in Figure 4

All hydrogen atoms except methanol are omitted for clarity

图 5 和图 6 显示了 $\mathrm{Cp} * \mathrm{Ru}(\mathrm{CO})\left(=\mathrm{SiMes}_{2}\right) \mathrm{SiMe}_{3}$ 中 硅配位体上的甲基连续被甲氧基取代的反应步骤. 图 5 中 $6 \mathrm{a}$ 到 $9 \mathrm{a}$ 为甲氧基化的第二步, 图 6 中 $9 \mathrm{a}$ 到 12a 为甲 氧基化的第三步. 甲氧基化的过程都由 1,2-甲基转移开 始, 经过甲醇的加成反应, 甲醇中羟基上的氢原子从氧 原子转移到与 $\mathrm{Ru}$ 相连的甲基上, 最终脱除甲烷生成甲 氧基化中间产物. 计算得到的自由能势能面图表明两步 甲氧基化过程在热力学上都是可行的.

如图 5 所示, 在第二步甲氧基的反应中, 配合物 6a 首先发生 1,2-甲基转移反应, 过渡态的活化能垒 $\left(\mathbf{T S}_{6 \mathrm{a}-7}\right)$ 为 $11.3 \mathrm{kcal} / \mathrm{mol}, \mathbf{T S}_{6 \mathrm{a}-7}$ 中 $\mathrm{Si}-\mathrm{C}, \mathrm{Ru}-\mathrm{C}$ 和 $\mathrm{Ru}-\mathrm{Si}$ 的距 离分别为 $2.415,2.302$ 和 $2.258 \AA$ (图 8). 此步反应生成中 间产物 7. 随着甲醇中的氧原子配位到硅原子上, 羟基 中的氢原子从氧原子转移到与 $\mathrm{Ru}$ 相连的甲基上, 生成 含有抓氢键的配合物 $\mathbf{9}$, 反应的活化能垒 $\left(\mathbf{T S}_{8-9}\right)$ 为 9.1 $\mathrm{kcal} / \mathrm{mol}, \mathbf{T S}_{8-9}$ 中 $\mathrm{O}-\mathrm{H}$ 和 $\mathrm{C}-\mathrm{H}$ 的距离分别为 1.180 和 $1.491 \AA$ \&. 最后, 配合物 9 中释放出 $\mathrm{CH}_{4}$, 放出 7.3 $\mathrm{kcal} / \mathrm{mol}$ 的能量, 生成中间产物 9a. 如图 6 所示, 第三步 甲氧基的反应机理跟第二步类似, 其中 1,2-甲基转移的 活化能垒 $\left(\mathbf{T S}_{9 \mathrm{a}-10}\right)$ 为 $9.9 \mathrm{kcal} / \mathrm{mol}$, 氢原子转移的活化能 垒 $\left(\mathbf{T S}_{11-12}\right)$ 为 $8.2 \mathrm{kcal} / \mathrm{mol}$. 随着 $\mathrm{CH}_{4}$ 的释放, 反应生成 完全甲氧基化的中间产物 12a, 最终异腈配位得到产物 13, 整个反应放热 $107.1 \mathrm{kcal} / \mathrm{mol}$.

\section{2 甲硅烷基(锗烯)钉配合物与甲醇的反应机理}

我们还研究了甲硅烷基(锗烯)钉配合物 $\mathrm{Cp} * \mathrm{Ru}-$
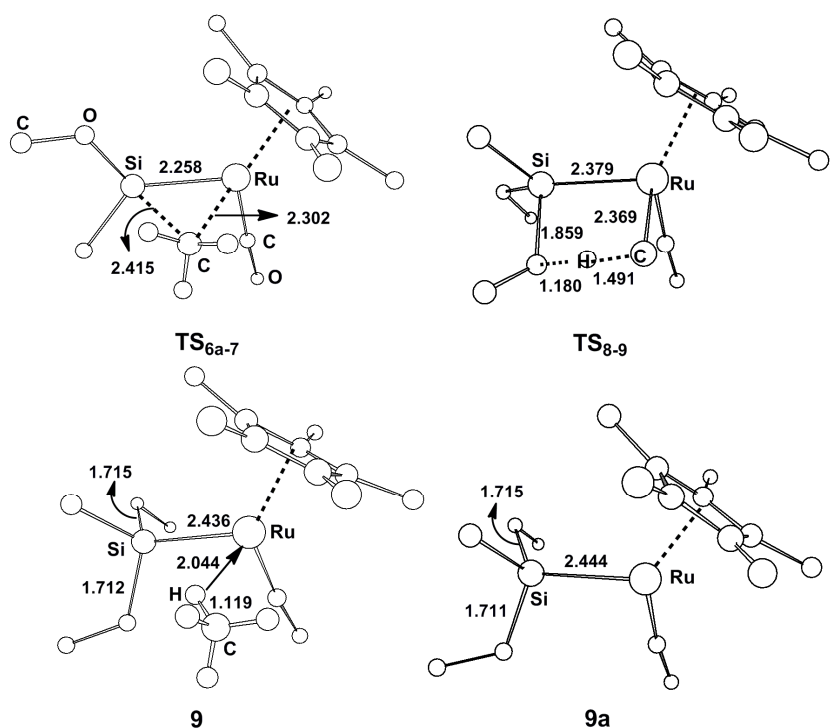

$\mathrm{TS}_{8-9}$

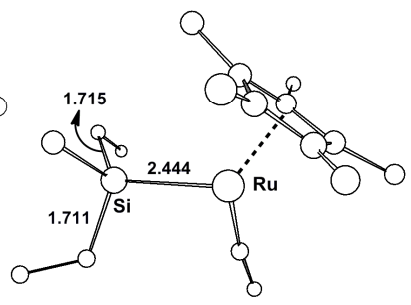

$9 a$

图 8 优化得到的如图 5 中所示的一些配合物的几何结构和键 长 $(\AA)$

Figure 8 Optimized structures with selected bond lengths $(\AA)$ for the species shown in Figure 5

All hydrogen atoms except methanol are omitted for clarity
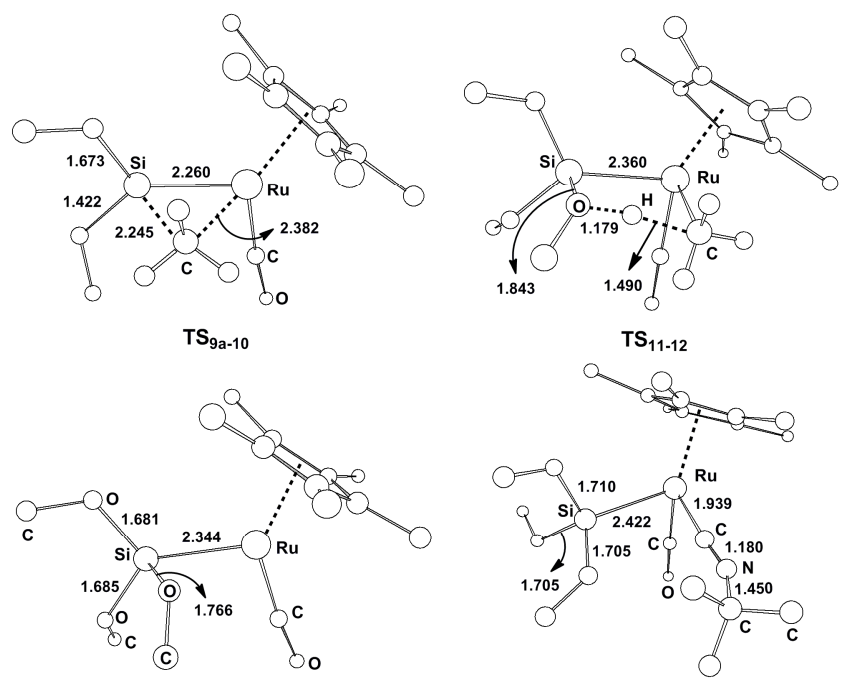

$12 a$

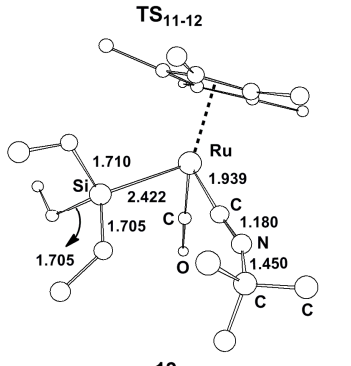

13

图 9 优化得到的如图 6 中所示的一些配合物的几何结构和键 长 $(\AA)$

Figure 9 Optimized structures with selected bond lengths $(\AA)$ for the species shown in Figure 6

All hydrogen atoms except methanol are omitted for clarity

(CO) $\left(=\mathrm{GeMes}_{2}\right) \mathrm{SiMe}_{3}$ (1a) 与甲醇的反应机理. 图 10 12 显示了该反应的自由能势能面图, 重要结构的几何 参数如图 13 所示.

该反应的势能面图与配合物 $\mathbf{1}$ 和甲醇反应的势能面 图相似. 反应机理也包含四个步骤: (1)甲醇和 $\mathrm{Ru}=\mathrm{Ge}$ 双键发生 1,2 加成, 消除 $\mathrm{Mes}_{2} \mathrm{Ge}(\mathrm{OMe}) \mathrm{H}$, 生成中间产物 $\mathrm{Cp} * \mathrm{Ru}(\mathrm{CO}) \mathrm{SiMe}_{3}$; (2) 甲硅烷基配体上的甲基通过 1,2-甲 


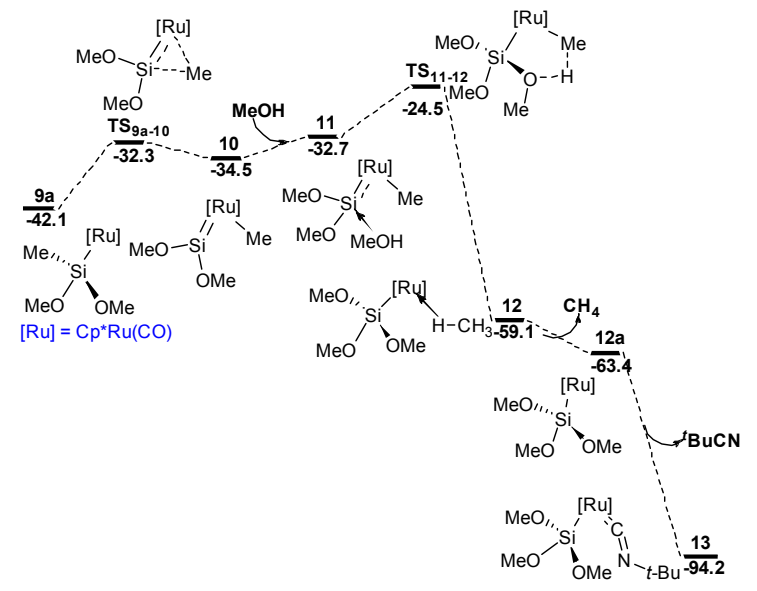

图 10 配合物 $1 \mathrm{a}$ 和甲醇反应中甲醇加成和甲硅烷基第一次甲 氧基化的自由能势能面(溶剂化自由能)

Figure 10 Free energy profiles of the first alkoxylation of the silyl ligand for the reaction of complex $\mathbf{1 a}$ with methanol

The solvation-corrected relative free energies are given in $\mathrm{kcal} / \mathrm{mol}$

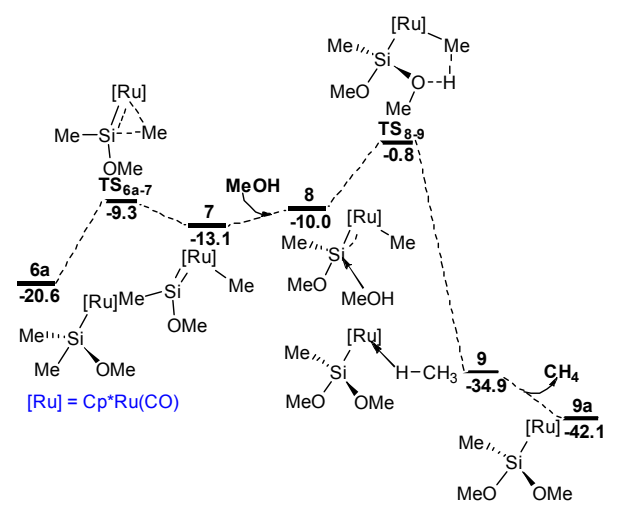

图 11 配合物 $1 \mathrm{a}$ 和甲醇反应中甲硅烷基第二次甲氧基化的自 由能势能面(溶剂化自由能)

Figure 11 Free energy profiles for the second alkoxylation of the silyl ligand for the reaction of complex 1a with methanol

The solvation-corrected relative free energies are given in $\mathrm{kcal} / \mathrm{mol}$

基的迁移方式从硅原子转移到 $\mathrm{Ru}$ 原子上, 再与甲醇发 生 1,2 加成反应, 释放甲烷后生成中间产物 $\mathrm{Cp} * \mathrm{Ru}(\mathrm{CO}) \mathrm{Si}(\mathrm{OMe}) \mathrm{Me}_{2} ;$; (3)重复步骤(2)两次, 得到完全 烷氧基化的产物 $\mathrm{Cp} * \mathrm{Ru}(\mathrm{CO}) \mathrm{Si}(\mathrm{OMe})_{3}$; (4)异腈配位后得 到最终产物 $\mathrm{Cp} * \mathrm{Ru}(\mathrm{CO})(\mathrm{CN} t-\mathrm{Bu}) \mathrm{Si}(\mathrm{OMe})_{3}$. 在这个反 应中, 决速步骤为甲醇与 $\mathrm{Ru}=\mathrm{Ge}$ 双键的 1,2 加成, 该能 垒 $\left(\mathbf{T S}_{1 \mathrm{a}-2 \mathrm{a}}\right)$ 达到 $31.8 \mathrm{kcal} / \mathrm{mol}$, 比硅烯钓化物 $\mathbf{1}$ 低 3.5 $\mathrm{kcal} / \mathrm{mol}$, 表明甲硅烷基(锗烯)钓配合物 $1 \mathbf{a}$ 与甲醇的反 应速率更快.

图 14 显示了过渡态 $\mathbf{T S}_{1-2}$ 和 $\mathbf{T S}_{1 \mathrm{a}-2 \mathrm{a}}$ 的自然电荷布居 分析. 计算结果表明 $\mathbf{T S}_{1-2}$ 中钓原子和硅原子的带电量 分别为 $-1.241 \mathrm{e}$ 和 $1.609 \mathrm{e}$. $\mathbf{T S}_{1 \mathrm{a}-2 \mathrm{a}}$ 中钓原子和锗原子分 别带电量分别为 $-1.280 \mathrm{e}$ 和 $1.654 \mathrm{e}$, 计算表明 $\mathbf{T S}_{1-2}$ 中的 $\mathrm{Ru}=\mathrm{Si}$ 双键和 $\mathbf{T S}_{1 \mathrm{a}-2 \mathrm{a}}$ 中的 $\mathrm{Ru}=\mathrm{Ge}$ 双键均以 $\mathrm{Ru}\left({ }^{\delta-}\right)$ -

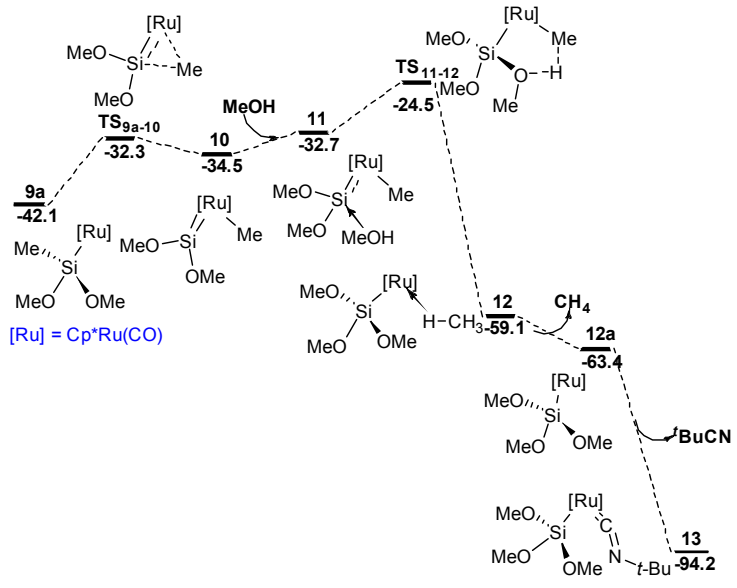

图 12 配合物 $1 \mathrm{a}$ 和甲醇反应中甲硅烷基第三次甲氧基化和异 腈配位的自由能势能面(溶剂化自由能)

Figure 12 Free energy profiles for the third alkoxylation of the silyl ligand and the coordination of isonitrile for the reaction of complex 1a with methanol

The solvation-corrected relative free energies are given in $\mathrm{kcal} / \mathrm{mol}$
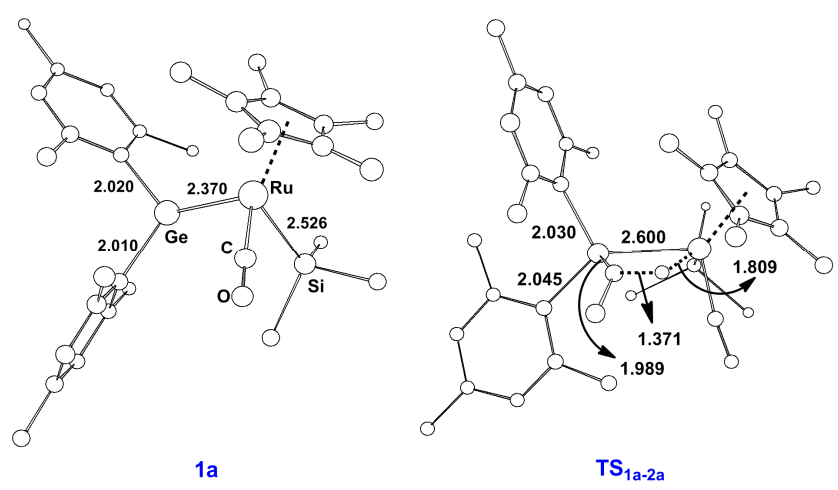

图 13 优化得到的如图 10 中所示的 $1 \mathbf{a}$ 和 $\mathbf{T S}_{1 \mathrm{a}-2 \mathrm{a}}$ 的几何结构 和键长 $(\AA)$

Figure 13 Optimized structures with selected bond lengths $(\AA)$ for the species 1a and $\mathbf{T S}_{1 \mathrm{a}-2 \mathrm{a}}$ shown in Figure 10

All hydrogen atoms except methanol are omitted for clarity

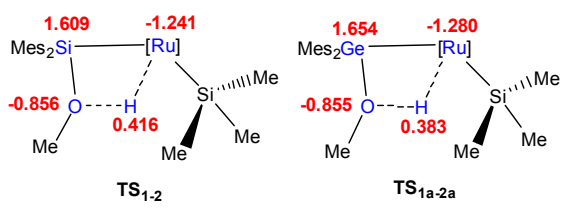

图 $14 \mathrm{TS}_{1-2}$ 和 $\mathrm{TS}_{1 \mathrm{a}-2 \mathrm{a}}$ 的自然电荷布居分析

Figure 14 The natural charge analysis of the transition state $\mathbf{T S}_{1-2}$ and $\mathbf{T S}_{1 \mathrm{a}-2 \mathrm{a}}$

$\mathrm{M}\left({ }^{\delta+}\right)$ 的形式存在, 钉原子也因而呈现出亲核性, 促使 甲醇中的氧原子配位到硅(锗)中心, 而氢原子从氧原子 转移到钉原子上. 此外, $\mathbf{T S}_{1 \mathrm{a}-2 \mathrm{a}}$ 中的钓中心比 $\mathbf{T S}_{1-2}$ 中的 钓中心带有更多的负电荷, 锗原子比硅原子带更多的正 电荷, 因而 $\mathrm{Ru}-\mathrm{Ge}$ 比 $\mathrm{Ru}-\mathrm{Si}$ 键极化程度更高, 这与我 们的计算结果相一致，在决速步 1,2-甲醇的加成反应中, 锗烯配合物的能垒要比硅烯配合物低. 


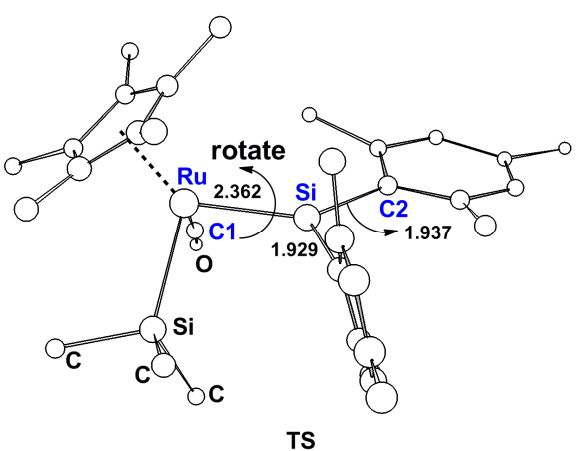

TS

图 15 优化得到的配合物 $\mathbf{1}$ 中 $\mathrm{Ru}=\mathrm{Si}$ 键旋转过渡态的几何结 构和键长 $(\AA)$

Figure 15 Optimized structures with selected bond lengths $(\AA)$ for the rotation transition state of complex 1 All hydrogen atoms are omitted for clarity

\section{3 配合物中 $\mathrm{Ru}=\mathrm{Si}$ 键的旋转}

在实验中, 通过对不同温度下核磁共振氢谱的研 究, 我们发现配合物 $\mathbf{1}$ 的 $\mathrm{Ru}=\mathrm{Si}$ 双键可以旋转, 相应的 活化参数为 $\Delta H=15(3) \mathrm{kcal} / \mathrm{mol}, \Delta G=16(6) \mathrm{kcal} / \mathrm{mol}$, $\Delta S=-4(5) \mathrm{cal} /(\mathrm{K} \cdot \mathrm{mol})$. 为了解配合物 1 中的 $\mathrm{Ru}=\mathrm{Si}$ 双键旋转, 我们对此步骤进行了计算, 优化的过渡态结 构如图 15 所示. 计算结果表明键旋转参数分别为 $\Delta H_{1}$ $=22.57 \mathrm{kcal} / \mathrm{mol}, \Delta G_{1}=24.95 \mathrm{kcal} / \mathrm{mol}, \Delta S_{1}=-7.99$ $\mathrm{cal} /(\mathrm{K} \cdot \mathrm{mol})$, 与实验结果相一致.

\section{2 结论}

在本文中, 我们运用 DFT 计算方法研究了甲硅烷 基(硅烯)钌配合物 $\mathrm{Cp} * \mathrm{Ru}(\mathrm{CO})\left(=\mathrm{SiMes}_{2}\right) \mathrm{SiMe}_{3}$ (1)和甲 硅烷基(锗烯)钌配合物 $\mathrm{Cp} * \mathrm{Ru}(\mathrm{CO})\left(=\mathrm{GeMes}_{2}\right) \mathrm{SiMe}_{3}$ (1a)分别与甲醇反应的机理. 在配合物 1 与甲醇的反应 过程中, 甲醇发生 1,2 加成的活化能垒为 $35.3 \mathrm{kcal} / \mathrm{mol}$, 是甲氧基转化过程中的决速步骤. 在配合物 $1 \mathrm{a}$ 与甲醇 的反应过程中, 最慢的步骤也是 1,2 加成, 所需能量是 $31.8 \mathrm{kcal} / \mathrm{mol}$. 通过对过渡态 $\mathbf{T S}_{\mathbf{1 - 2}}$ 和 $\mathbf{T S}_{\mathbf{1 a}-\mathbf{2} \mathbf{a}}$ 的自然电荷 布居分析, 可以发现 $\mathrm{Ru}=\mathrm{M}(\mathrm{M}$ 为 $\mathrm{Si}$ 或 $\mathrm{Ge})$ 双键呈现出 $\mathrm{Ru}\left({ }^{\delta-}\right)-\mathrm{M}\left({ }^{\delta+}\right)$ 的极化方式. 与 $\mathbf{T S}_{\mathbf{1 - 2}}$ 相比, $\mathbf{T S}_{\mathbf{1 a - 2}}$ 中的钓 原子带有更多的负电荷, 而锗原子则带有更多的正电 荷, 这有利于氢原子的转移, 降低了自由能势垒. 我们 的计算结果为研究过渡金属硅烯和锗烯配合物，提供了 重要的理论基础.

\section{3 计算方法}

采用杂化 Becke3LYP (B3LYP)的计算方法, 我们对 反应中涉及的所有反应物、中间产物、过渡态和产物的 几何结构进行了全面优化 ${ }^{[13]}$. 我们的前期工作 ${ }^{[11,14]}$ 和其 它涉及过渡金属甲硅烷(硅烯)钉配合物理论研究已经证
实了所选方法的可靠性. 通常情况下我们使用与前期研 究相一致的基组 ${ }^{[15]}$. 在 DFT 计算中, 我们对 $\mathrm{H}, \mathrm{C}, \mathrm{N}$ 和 $\mathrm{O}$ 原子采用 6-31G(d)基组. 而对 $\mathrm{Si}, \mathrm{Ge}$ 和 $\mathrm{Ru}$ 原子采用 Hay 和 Wadt 发展的带有效核势的双 $\zeta$ 价基组 (LANL2DZ) 来描述 ${ }^{[16]}$, 并且对这些原子又添加了极化 函数, $\operatorname{Ru}\left(\zeta_{\mathrm{f}}\right)=1.235, \operatorname{Si}\left(\zeta_{\mathrm{d}}\right)=0.262, \mathrm{Ge}\left(\zeta_{\mathrm{d}}\right)=0.246^{[17,18]}$.

通过频率分析计算, 可以获得 Gibbs 自由能和零点 能(ZPE), 表征势能面上的稳定点为最小值(零虚频)或 过渡态(一个虚频). 通过过渡态的内禀反应坐标计算 (IRC) 来确认这种结构确实连接相关的两个极小值 ${ }^{[19]}$. $\mathrm{NBO}$ 方法被用于自然电荷布居分析，以获取每个原子 的电荷 ${ }^{[20]}$.

考虑到溶剂的影响，采用类导体极化连续模型 $(\mathrm{CPCM})$ 的 $\mathrm{UAHF}$ 半径 ${ }^{[21]}$, 用于所有优化产物的单点能 量计算. 根据实验的反应条件, 所用的溶剂是苯. 在包 含势能面的所有图中，显示的是溶剂条件下的相对自由 能，所有的计算在 Gaussian09 中完成 ${ }^{[22]}$.

\section{References}

[1] Straus, D. A.; Zhang, C.; Quimbita, G. E.; Grumbine, S. D.; Heyn, R. H.; Tilley, T. D.; Rheingold, A. L.; Geib, S. J. J. Am. Chem. Soc. 1990, 112, 2673.

[2] Straus, D. A.; Tilley, T. D. J. Am. Chem. Soc. 1987, 109, 5872.

[3] Waterman, R.; Hayes, P. G.; Tilley, T. D. Acc. Chem. Res. 2007, 40,712 .

[4] (a) Calimano, E.; Tilley, T. D. J. Am. Chem. Soc. 2008, 130, 9226. (b) Calimano, E.; Tilley, T. D. J. Am. Chem. Soc. 2009, 131, 11161. (c) Calimano, E.; Tilley, T. D. Organometallics 2010, 29, 1680. (d) Fasulo, M. E.; Glaser, P. B.; Tilley, T. D. Organometallics 2011, 30, 5524.

(e) Fasulo, M. E.; Tilley, T. D. Organometallics 2012, 31, 5049. (f) Fasulo, M. E.; Lipke, M. C.; Tilley, T. D. Chem. Sci. 2013, 4, 3882.

[5] Ueno, K.; Tobita, H.; Shimoi, M.; Ogino, H. J. Am. Chem. Soc. 1988, 110, 4092

[6] (a) Ogino, H. Chem. Rec. 2002, 2, 291. (b) Okazaki, M.; Tobita, H.; Ogino, H. Dalton Trans. 2003, 493.

[7] Watanabe, T.; Hashimoto, H.; Tobita, H. Angew. Chem., Int. Ed. 2004, 43, 218

[8] Watanabe, T.; Hashimoto, H.; Tobita, H. J. Am. Chem. Soc. 2006, $128,2176$.

[9] Watanabe, T.; Hashimoto, H.; Tobita, H. J. Am. Chem. Soc. 2007, $129,11338$.

[10] Ochiai, M.; Hashimoto, H.; Tobita, H. Organometallics 2012, 31, 527.

[11] Xie, H.; Lin, Z. Organometallics 2014, 33, 892.

[12] Hashimoto, H.; Sato, J.; Tobita, H. Organometallics 2009, 28 , 3963.

[13] (a) Becke, A. D. Phys. Rev. A 1988, 38, 3098 (b) Lee, C.; Yang, W.; Parr, R. G. Phys. Rev. B 1988, 37, 785.

(c) Becke, A. D. J. Chem. Phys. 1993, 98, 5648

[14] (a) Arnold Jr, F. P. Organometallics 1999, 18, 4800.

(b) Zhang, X. H.; Chung, L. W.; Lin, Z.; Wu, Y. D. J. Org. Chem. 2008, 73, 820 .

(c) Chung, L. W.; Wu, Y.-D.; Trost, B. M.; Ball, Z. T. A. J. Am. 
Chem. Soc. 2003, 125,11578

[15] (a) Xie, H.; Lin, F.; Yang, L.; Chen, X.; Ye, X.; Tian, X.; Lei, Q.; Fang, W. J. Organomet. Chem. 2013, 745-746, 417.

(b) Xie, H.; Lin, F.; Lei, Q.; Fang, W. Organometallics 2013, 32, 6957.

(c) Ye, X.; Yang, L.; Li, Y.; Huang, J.; Zhou, L.; Lei, Q.; Fang, W.; Xie, H. Eur. J. Inorg. Chem. 2014, 1502.

[16] (a) Check, C. E.; Faust, T. O.; Bailey, J. M.; Wright, B. J.; Gilbert, T. M.; Sunderlin, L. S. J. Phys. Chem. A 2001, 105, 8111.

(b) Hay, P. J.; Wadt, W. R. J. Chem. Phys. 1985, 82, 299.

[17] Höllwarth, A.; Böhme, M.; Dapprich, S.; Ehlers, A. W.; Gobbi, A.; Jonas, V.; Köhler, K. F.; Stegmann, R.; Veldkamp, A.; Frenking, G. Chem. Phys. Lett. 1993, 208, 237.

[18] Ehlers, A. W.; Böhme, M.; Dapprich, S.; Gobbi, A.; Höllwarth, A.; Jonas, V.; Köhler, K. F.; Stegmann, R.; Veldkamp, A.; Frenking, G. Chem. Phys. Lett. 1993, 208, 111.

[19] (a) Fukui, K. J. Phys. Chem. 1970, 74, 4161.

(b) Fukui, K. Acc. Chem. Res. 1981, 14, 363.

[20] (a) Reed, A. E.; Weinstock, R. B.; Weinhold, F. J. Chem. Phys. 1985, 83, 735 .

(b) Reed, A. E.; Weinhold, F. J. Chem. Phys. 1985, 83, 1736.

(c) Reed, A. E.; Curtiss, L. A.; Weinhold, F. Chem. Rev. 1988, 88,
899.

[21] (a) Barone V.; Cossi, M. J. Phys. Chem. A 1998, 102, 1995.

(b) Cossi, M.; Rega, N.; Scalmani, G.; Barone, V. J. Comput. Chem. 2003, 24, 669

[22] Frisch, M. J.; Trucks, G. W.; Schlegel, H. B.; Scuseria, G. E.; Robb, M. A.; Cheeseman, J. R.; Scalmani, G.; Barone, V.; Mennucci, B.; Petersson, G. A.; Nakatsuji, H.; Caricato, M.; Li, X.; Hratchian, H. P.; Izmaylov, A. F.; Bloino, J.; Zheng, G.; Sonnenberg, J. L.; Hada, M.; Ehara, M.; Toyota, K.; Fukuda, R.; Hasegawa, J.; Ishida, M.; Nakajima, T.; Honda, Y.; Kitao, O.; Nakai, H.; Vreven, T.; Montgomery, J. A.; Peralta, Jr. J. E.; Ogliaro, F.; Bearpark, M.; Heyd, J. J.; Brothers, E.; Kudin, K. N.; Staroverov, V. N.; Kobayashi, R.; Normand, J.; Raghavachari, K.; Rendell, A.; Burant, J. C.; Iyengar, S. S.; Tomasi, J.; Cossi, M.; Rega, N.; Millam, J. M.; Klene, M.; Knox, J. E.; Cross, J. B.; Bakken, V.; Adamo, C.; Jaramillo, J.; Gomperts, R.; Stratmann, R. E.; Yazyev, O.; Austin, A. J.; Cammi, R.; Pomelli, C.; Ochterski, J. W.; Martin, R. L.; Morokuma, K.; Zakrzewski, V. G.; Voth, G. A.; Salvador, P.; Dannenberg, J. J.; Dapprich, S.; Daniels, A. D.; Farkas, O.; Foresman, J. B.; Ortiz, J. V.; Cioslowski, J.; Fox, D. J. Gaussian 09, Revision A.1, Gaussian, Inc., Wallingford CT, 2009.

$(\mathrm{Lu}, \mathrm{Y}$ ) 